Document downloaded from:

http://hdl.handle.net/10251/153679

This paper must be cited as:

Cabrero Antonino, JR.; Adam-Ortiz, R.; Wärnå, J.; Murzin, DY.; Beller, M. (2018). Reductive $\mathrm{N}$-methylation of amines using dimethyl carbonate and molecular hydrogen: Mechanistic insights through kinetic modelling. Chemical Engineering Journal. 351:1129-1136. https://doi.org/10.1016/j.cej.2018.06.174

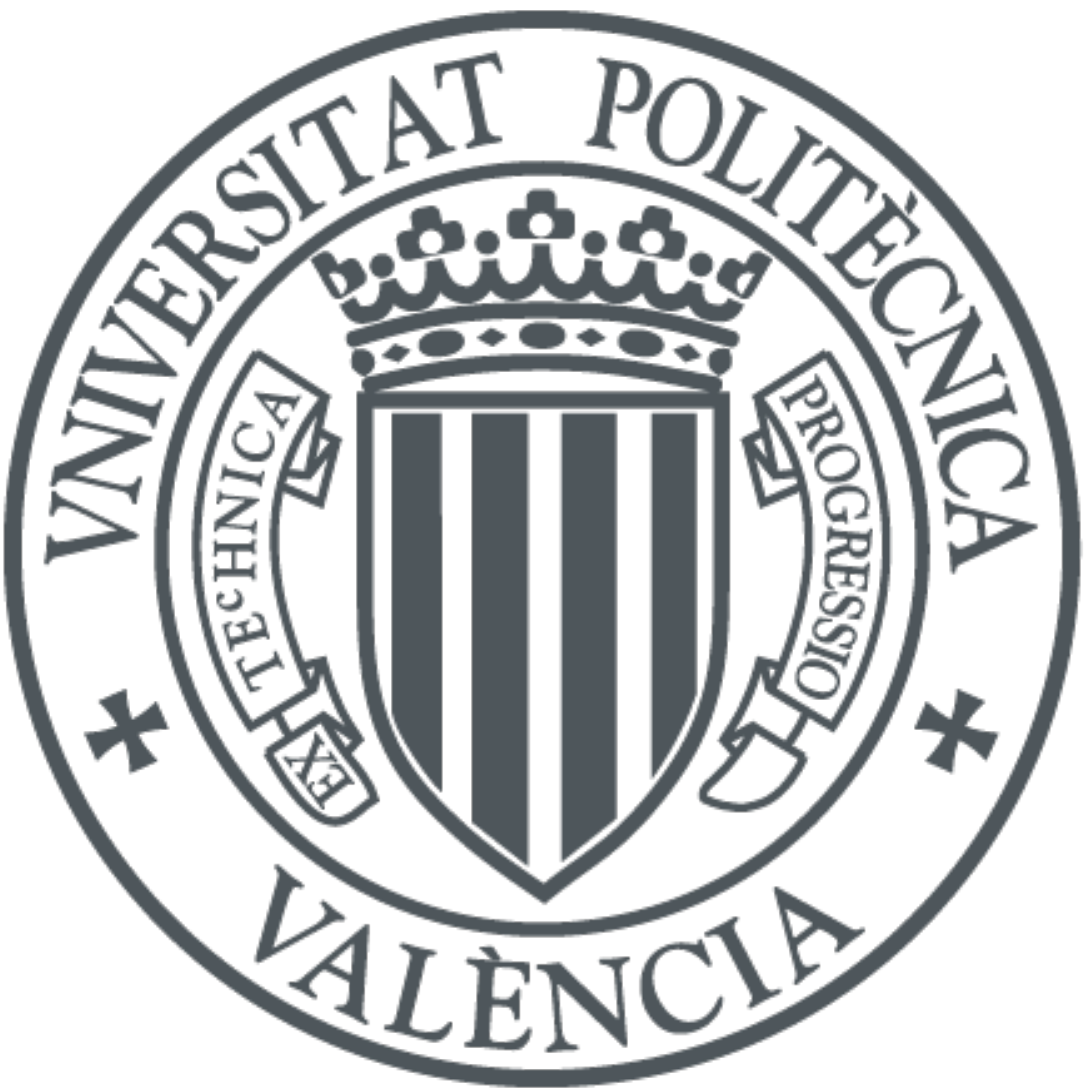

The final publication is available at

https://doi.org/10.1016/j.cej.2018.06.174

Copyright Elsevier

Additional Information 


\title{
Reductive N-methylation of amines using dimethyl carbonate and molecular hydrogen: mechanistic insights through kinetic modelling
}

\author{
Jose R. Cabrero-Antonino ${ }^{1}$, Rosa Adam ${ }^{1}$, Johan Wärnå2, \\ Dmitry Yu. Murzin ${ }^{2 *}$, Matthias Beller ${ }^{1}$
}

${ }^{1}$ Leibniz-Institut für Katalyse e.V. an der Universität Rostock, Rostock, Germany

${ }^{2}$ Åbo Akademi University, Åbo/Turku, Finland

corresponding author: E-mail: dmurzin@abo.fi

\begin{abstract}
Kinetic analysis of ruthenium-catalyzed reductive N-methylation of amines using dimethyl carbonate as $\mathrm{C} 1$ source and molecular hydrogen has been performed. Kinetic equations have been derived and kinetic modelling has been performed for experimental data generated previously at a constant hydrogen pressure as well as for additional experiments performed at different hydrogen pressures. The study has revealed interesting kinetic features related to an induction period strongly influenced by temperature. A kinetic model has been proposed based on advanced reaction mechanism featuring transformation between different type of catalytic species and inactivation of them during the reaction. Kinetic modelling was done for all data sets together showing excellent correspondence between calculations and experiments.
\end{abstract}




\section{Introduction}

Kinetic analysis of complex non-catalytic and catalytic organic reactions has become a tool more and more utilized in academia and industry [1-5]. This kind of analysis has acquired relevance in chemical reaction engineering [6-10], where rather complex mechanisms are rigorously treated using numerical data fitting. In particular, for catalysis, a kinetic phenomenon, elucidation of kinetics is an essential part. Typically, experimental data are collected as a function of the parameters considered important for the reaction, i.e. concentrations, temperature, pressures, $\mathrm{pH}$, catalyst concentration, volume, etc. Thereafter, a reaction mechanism is proposed based on mechanistic, spectroscopic and kinetic data, estimating the rate constants by regression analysis. Finally, the adequacy of the model is judged based on criteria such as residual sums of squares and parameter significance.

Classical methods of organic chemistry typically provide only a snapshot of a particular reaction (conversion and selectivity after a certain reaction time). In fact, monitoring reaction kinetics of catalytic reactions with a synthetic interest is not a commonly used tool. However, the kinetic information, together with various spectroscopy and computational methods, could provide a substantial amount of mechanistic information useful in the improvement of synthetic methodologies.

The selective N-methylation of amines is a frequently used reaction in organic synthesis, affording valuable compounds used as drugs, agrochemicals or dyes [11-13]. From an industrial perspective, the preferred methylation agents are formaldehyde, using the Eschweiler-Clarke methodology [13] and methanol, employing zeolites as dehydration catalysts.[14] An 
interesting alternative to these methodologies would be the use of dimethyl carbonate (DMC) in the presence of a reducing agent, as DMC is a non-toxic, safe and biodegradable compound which is currently used in the industry. Moreover, among the currently used protocols for the synthesis of DMC, an emerging one is based on the reaction of carbon dioxide with methanol [15-17].

Traditionally, the employment of DMC as a methylating agent involved a $\mathrm{B}_{\mathrm{AL}} 2$ mechanism, in which the methyl carbon of the methoxy group acts as the electrophile.[18, 19] This methodology presents drawbacks, such as using high reaction temperatures $\left(>160^{\circ} \mathrm{C}\right)$ to avoid carbamoylation processes and having a limited substrate scope. In contrast, selective Nmethylation of amines with dialkyl carbonates and a reducing agent, where the $\mathrm{C}=\mathrm{O}$ moiety is the one used for the methylation, has also been described. Former examples of this reaction employed as catalysts a photo-activated iron-complex [20] or a platinum based complex [21] in the presence of hydrosilanes. The generation of large amounts of waste due to the use of an excess of hydrosilanes, and the limited substrate scope (only secondary amines were reactive), were important limitations of these protocols.

Recently, the first N-methylation of amines using DMC and molecular hydrogen has been described employing the $\left[\mathrm{Ru} / \mathrm{Triphos} / \mathrm{HNTf}_{2}\right]$ system as catalyst (Scheme 1) [22-24] where Triphos stands for 1,1,1-tris(diphenylphosphinomethyl)ethane [11]. The presence of the acid $\mathrm{HNTf}_{2}$ as co-catalyst (2.5 eq respect to the Ru loading) was key for the catalytic activity of the system. A mechanistic explanation for the role of the additive implies the formation of the $[\mathrm{Ru}(\text { Triphos })]^{2+}$ cation species stabilized by the weakly coordinating ${ }^{-}\left[\mathrm{NTf}_{2}\right]$ (bis(trifluoromethanesulfonyl)imide) [25]. This reductive N-methylation protocol, using dimethyl carbonate as $\mathrm{C} 1$ source and hydrogen as a reducing agent, was successfully applied for a wide range of primary and secondary aromatic and aliphatic amines. In addition, kinetic profiles for the formation of N-methylaniline $\mathbf{2}$ and N,N-dimethylaniline $\mathbf{3}$ under 60 bar of $\mathrm{H}_{2}$ 
at several reaction temperatures $\left(130,140,150\right.$ and $\left.160^{\circ} \mathrm{C}\right)$ were reported. A tentative reaction mechanism for the $\mathrm{N}$-methylation of aromatic amines using this Ru-catalyzed methodology was proposed.

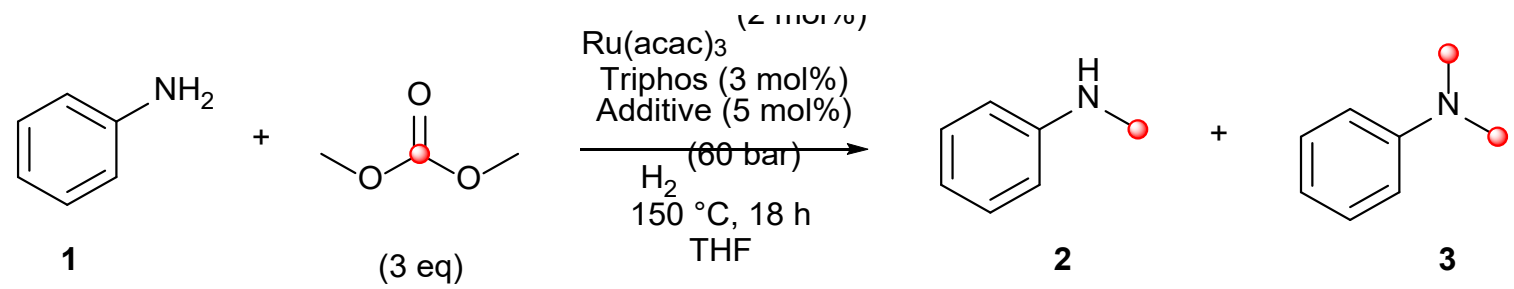

Scheme 1. Reductive N-methylation of aniline 1 using dimethyl carbonate (DMC) and molecular hydrogen catalyzed by $\left[\mathrm{Ru} / \mathrm{Triphos} / \mathrm{HNTf}_{2}\right]$ system.

Interestingly, the experimental data reported in this work [11] clearly display a S-shape behavior for the disappearance of aniline at low temperatures. In order to reveal mechanistic aspects of this reaction and to propose a rate equation capable to fit the experimental data, in the current work kinetic equations have been derived and kinetic modelling has been performed for N-methylation of aniline 1 with $\mathrm{DMC}$ and molecular hydrogen. Moreover, additional experiments have been done at different hydrogen pressures to reveal the influence of the molecular hydrogen on the induction period as well as on the main reaction per se.

\section{Experimental}

\section{Kinetic study for $N$-methylation of aniline}

A $100 \mathrm{~mL}$ glass inlet containing a stirring bar was sequentially charged with aniline 1 (279.6 $\mu \mathrm{L}, 3.0 \mathrm{mmol})$, n-hexadecane $(250.0 \mathrm{mg})$ as an internal standard, $\mathrm{Ru}(\mathrm{acac})_{3}(24.1 \mathrm{mg}, 0.06$ mmol, $2 \mathrm{~mol} \%$ ), Triphos (58.5 mg, $0.09 \mathrm{mmol}, 3 \mathrm{~mol} \%$ ), THF (12 mL) as solvent, dimethyl carbonate $(868.2 \mu \mathrm{L}, 9.0 \mathrm{mmol}, 3$ eq. $)$ and a freshly prepared $0.2 \mathrm{M}$ THF solution of the cocatalyst triflimide HNTf2 $(750.0 \mu \mathrm{L}, 0.15 \mathrm{mmol}, 5 \mathrm{~mol} \%)$. Afterwards, the reaction vial was then placed into a $100 \mathrm{~mL}$ autoclave. Once sealed, the autoclave was purged three times with 30 bar of hydrogen, then pressurized to the corresponding reaction pressure (10-60 bar) and 
placed into an aluminium block, which was preheated at $130-160^{\circ} \mathrm{C}$. Periodically, aliquots of $200 \mu \mathrm{L}$ were taken at different times of reaction, diluted with ethyl acetate and analysed by GCFID, HP 6890 with FID detector, column HP5 $30 \mathrm{~m}$ x $250 \mathrm{~mm} \times 0.25 \mu \mathrm{m}$.

\section{Kinetic modelling}

In kinetic modelling, generation equations for the reactants and products were solved simultaneously for all data sets obtained at different pressures of hydrogen and temperature levels. In the estimation of the kinetic parameters, the residual sum of squares (Q) was minimized:

$Q=\sum_{k}\left(c_{\exp , t}-c_{t}\right)^{2}$

where 'exp' refers to the experimental data and $c$ the concentrations predicted by the model. The underlying differential equations were solved by a backward difference method implemented in a stiff ODE-solver during the parameter estimation. A Levenberg-Marquardt algorithm was used in the minimization of the objective function. The regression software [23] was used in the computations. The results were checked by standard statistical analysis as well as by the degree of explanation $\left(\mathrm{R}^{2}\right)$ defined by

$$
R^{2}=1-\frac{\Sigma\left(c_{i \exp , t}-c_{i, t}\right)^{2}}{\sum\left(c_{i \exp , t}-c_{i a v, t}\right)^{2}}
$$

The degree of explanation compares the actual model with the simplest possible model, i.e. description of the data by average values of the concentrations.

\section{Results and Discussion}

\section{Kinetic data}

Kinetic data for the N-methylation of aniline 1 with DMC at 60 bar of hydrogen and using different reaction temperatures, were already reported [11]. In addition, yield/time kinetic 
profiles were performed at $150^{\circ} \mathrm{C}$ using several hydrogen pressures (Figure 1). An analysis of these data shows that, at certain conditions, there is a clear induction period, where the reaction rate is rather low, since concentration of the starting substrate does not change. Thereafter, there is a rate acceleration, which can be related with the formation of a catalytically active species. As can be deduced from Figure 1b, the induction period exhibits a dependence on hydrogen pressure, as it is not observed at the higher pressure of 60 bar.

Figure 1a, as well as previously reported kinetic profiles [11], is typical for consecutive reactions, with initially formed compound $\mathbf{2}$ undergoing further transformations. Figure 1c displays the dependence of the product yields on conversion for a particular experiment at $150^{\circ} \mathrm{C}$ and 60 bar, illustrating that the monomethylated compound 2 passes through a maximum at ca. $50 \%$ conversion. It should be noted that the mass balance is not completely closed. This is related to formation of $\mathrm{N}$-phenylpyrrolidine from the acid-catalyzed ring opening reaction between aniline and THF (typically $2-5 \%$ is always detected). Moreover N-ethyl aniline can be generated from aniline and traces of ethanol reacting by so-called hydrogen borrowing reaction (detected in 1-2\%).
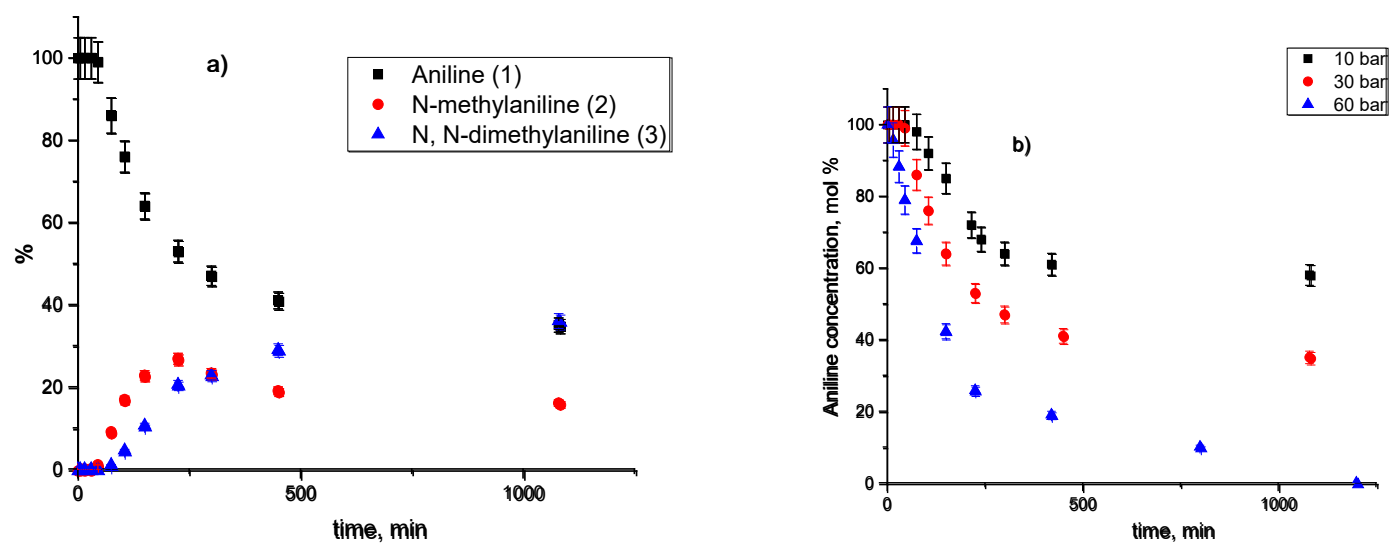

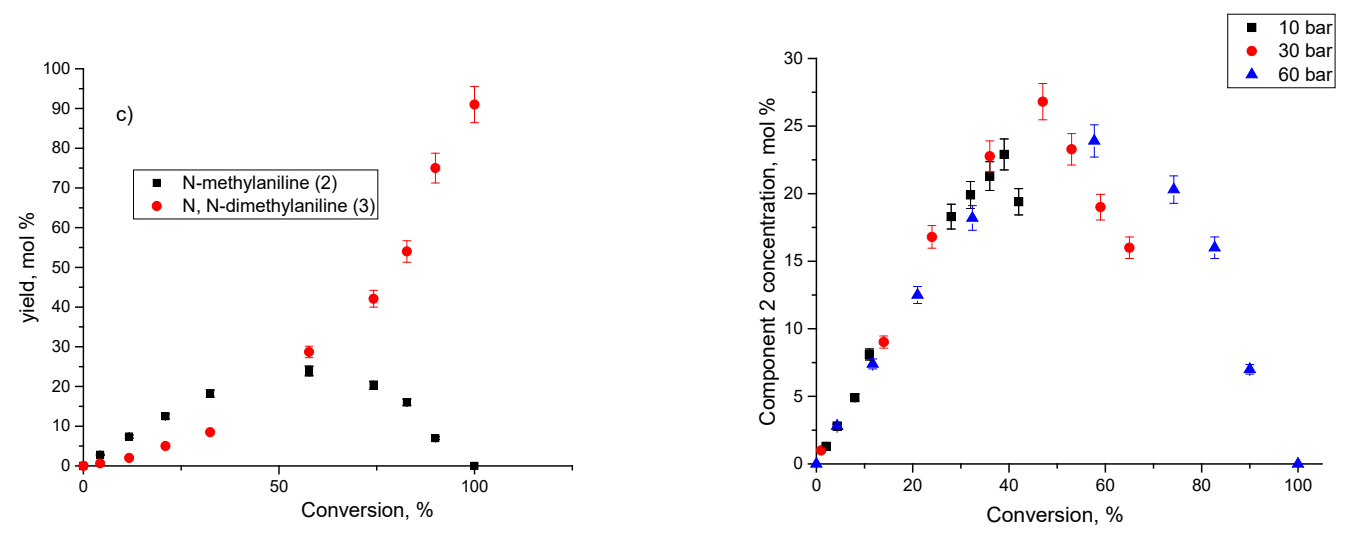

Figure 1. N-methylation of aniline 1 using dimethyl carbonate (3 eq) and molecular hydrogen at $150^{\circ} \mathrm{C}$ : a) kinetic profiles at $30 \mathrm{bar}, \mathrm{b}$ ) aniline concentration at different hydrogen pressure, c) yield of products $\mathbf{2}$ and $\mathbf{3} v s$ conversion of $\mathbf{1}$ at $60 \mathrm{bar}, \mathrm{d}$ ) yield of product $\mathbf{2}$ vs conversion at different hydrogen pressures. Figure contains error bars corresponding to $5 \%$.

From the reaction network viewpoint it is important to understand if selectivity dependence on conversion has any variation with hydrogen pressure. Figure $1 \mathrm{~d}$ convincingly demonstrates that in the consecutive alkylation of aniline 1 with DMC the yield towards the intermediate product is almost the same at least until the maximum is reached. Few experimental points above the maxima for 10 and 30 bar prevent from a conclusive statement about influence of hydrogen on the reaction route of further alkylation of the monomethylated product $\mathbf{2}$. This point will be addressed in the following text.

\section{Kinetic model}

Some sort of an induction period visible in Figure 1 and also seen in a very pronounced way in $\mathrm{N}$-methylation reactions in the low temperature region (Figure 2) can be generally explained by the concept of active site generation during the reaction. A similar concept has been used throughout the years for describing experimental data in various hydrogenation and hydrodeoxygenation reactions $[27,28]$. 


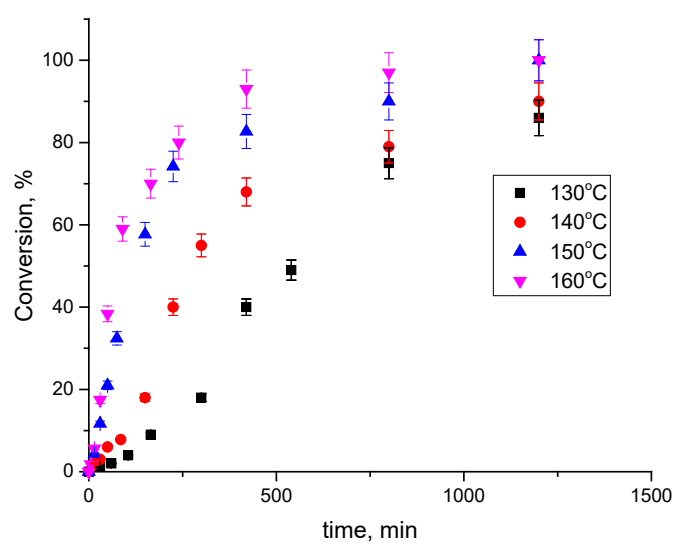

Figure 2. Conversion of aniline 1 vs reaction time in its N-methylation reaction using dimethyl carbonate and molecular hydrogen at different temperatures.

Transformation of compound $\mathbf{2}$ into compound $\mathbf{3}$ is not instantaneous, requiring presence of ligands and hydrogen. Another interesting feature of the reaction mechanism is catalyst deactivation visible in a most pronounced way in Figure $1 \mathrm{~b}$ for experiments conducted at 10 and 30 bar. At the moment based on the available data it is difficult to assess the exact nature of the inactive species.

Formation of $\left[(\text { Triphos }) \mathrm{RuH}(\mathrm{CO})_{2}\right]^{+}$as a nonreactive complex was proposed by Leitner and coworkers [25] using the same catalytic system in hydrogenation of $\mathrm{CO}_{2}$.

Further support for involvement of two difference sites comes from structure-activity studies. Hammett plots shown in Supporting Information of [11] and redrawn here illustrate an upward concave behaviour (Figure 3) apart from the case of fluorine as a substituent. 


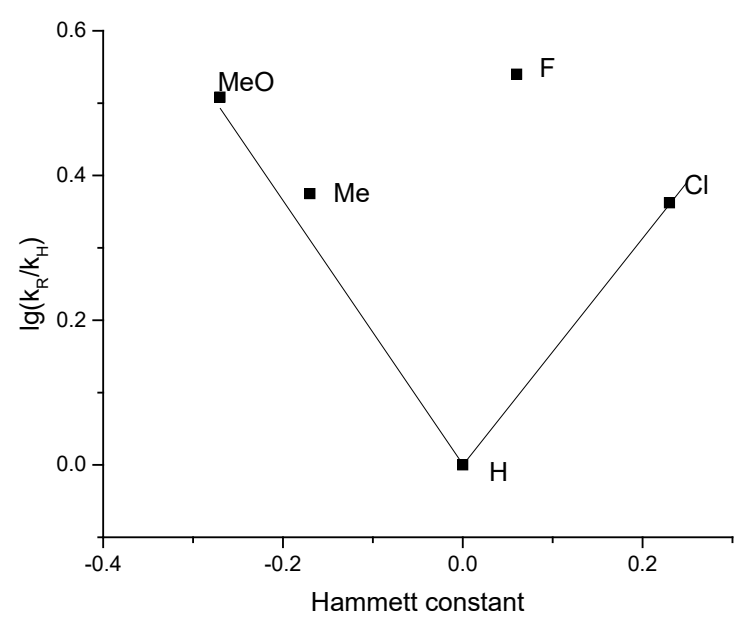

Figure 3. Hammett plot for the initial data of the N-methylation reaction of aniline $\mathbf{1}$ and different para-substituted derivatives 1-Me, 1-OMe, 1-F and 1-Cl with DMC and molecular hydrogen.

In general, non-linear Hammett plots with upward and downward concave behaviour have been reported in the literature [29-35]. While concave downwards plots can be explained by a complex reaction mechanism composed of a reversible and a subsequent irreversible step [36] upwards concave Hammett plots cannot be explained by changing the rate determining step in a multistep mechanism [37]. A complete change in the reaction mechanism [36] should be invoked for cases when electron donating groups are replaced by electron accepting ones.

In fact as shown in [37] the concept of a complete change in the reaction mechanism (with "on"-“off" point at $\mathrm{R}=\mathrm{H}$ ) should not be necessarily used. From the mathematical viewpoint simultaneous presence of two reaction pathways acting in different directions upon variation of the substituent can be sufficiently well described by the following equation

$$
r_{\sigma}=\left(k _ { I } ^ { 0 } 1 0 ^ { \rho _ { I } \sigma } f _ { I } \left(C_{i)}+k_{I I}^{0} 10^{\rho_{I I} \sigma} f_{I I}\left(C_{i)}\right) c_{c a t}\right.\right.
$$

This equation consists of two terms each corresponding to a distinct pathway (I or II) and reflecting different concentration dependences $\left.\left(f_{I}\left(C_{i}\right) ; f_{I I}\left(C_{i}\right)\right)\right)$ ). The reaction parameters $\rho_{I}$ and $\rho_{I I}$ should have opposite signs to account for the upward concave behaviour of the rate or apparent rate constant $v s$ substituent (Hammett) constant $\sigma$. When different signs of reaction 
constants $\rho_{I}$ and $\rho_{I I}$ contradict with the chemical nature of reactions present in a system displaying upward concave behaviour an alternative explanation of concave-upward was proposed [37]. It relies on the suggestion that at least two types of sites with different reactivity are present in the reaction milieu with the reaction itself proceeding through the same catalytic cycle.

Mathematically speaking, this suggestion results in the same description of the upward concave behavior as the concept of two routes. The kinetic equation comprises two terms each containing catalyst concentration of a particular type

$$
r_{\sigma}=r_{1} c_{c a t_{1}}+r_{2} c_{c a t_{2}}
$$

In eq. (4) $r_{1}$ and $r_{2}$ correspond to the rates for the first and the second type of catalytic species respectively with concentrations $c_{c a_{1}}$ and $c_{c a t_{2}}$. Such concept of two types of catalytic sites possessing different reactivity following, however, the same reaction mechanism was able of explaining an upward concave Hammett plot [37]. This theoretical explanation along with experimental data demonstrating an induction period in the methylation of aniline $\mathbf{1}$ with DMC provides a strong argument in favor of using a concept of two sites in the current work as well. The reaction mechanism comprising presence of two type of catalytic species in the reaction milieu as well as catalyst inactivation is presented in Figure 4. Species of A type are gradually transformed into species B, with which the catalytic reaction hand is faster. On the other hand, species of type B undergo inactivation resulting in some instances in a complete loss of activity. 


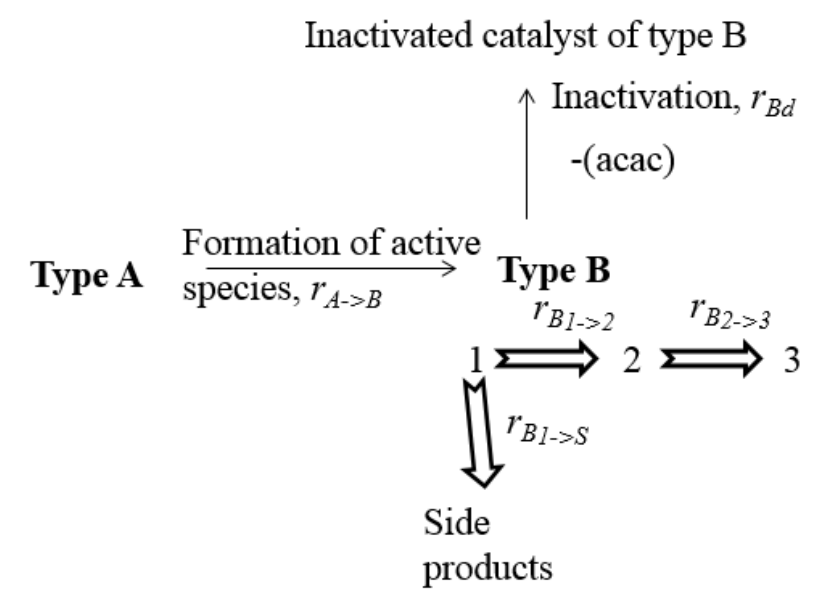

Figure 4. Reaction network involving different types of catalytic species.

While in general the reaction can proceed with both catalytic species A and B, preliminary kinetic modelling has demonstrated that reactions with species A can be neglected from the numerical viewpoint for reductive methylation of aniline. An argument in favor of this approach is related to a very clear delay with the reaction initiation at low pressure and low temperature. At the starting point of the reaction the active species of type B are formed. Preliminary attempts to use a simple approach of the first order kinetics for this transformation failed to explain experimental data. Observed pronounced induction period is thus a strong evidence that transformation of the type A sites to type B is more complex.

Quite often a concept of in-situ generation of "homogeneous" metal nanoclusters from the corresponding mononuclear metal precursors is used to explain dynamic nature of catalysis in cross-coupling and Heck reactions [38]. There are no, however, mechanistic reasons or experimental evidences for formation of such species, thus such concept will not be pursued further.

\section{Derivation of kinetic equations}

From the kinetic viewpoint explanation of the rate acceleration while reaction is progressing requires a suggestion that first A gives species of type B'. The latter species reacts with A finally 
resulting in active species of B type. Conceptually such active species can be either dimers according to $\mathrm{A}+\mathrm{B}^{\prime}->\mathrm{B}$ or monomers formally corresponding to the reaction $\mathrm{A}+\mathrm{B}$ ' $->\mathrm{B}+\mathrm{A}$. Formation of dimeric species was often reported in the literature for various reactions catalyzed by organometallics [39-41] and even discussed [25] in connection with the catalysts used in reductive methylation [11]. Experimental evidences based on various spectroscopy [25] are, however, favoring monomeric species as catalytic ones, thus only this more chemically relevant case will be described below. It should be noted that from the mathematical viewpoint both approaches give rather similar results. It is not possible, therefore, to discriminate between formation of monomeric and dimeric catalytically active species based exclusively on modelling of experimental data reported in [11] and additionally generated in the current work. In the mathematic treatment of the present work the concentration of catalytic species with time can be expressed

$$
\frac{d c_{c a t_{A}}}{d t}=-r_{A \rightarrow B^{\prime}}-r_{A \rightarrow B}+r_{A \rightarrow B} ; \frac{d c_{c a t_{B}}}{d t}=r_{A \rightarrow B}-r_{B d} ; \frac{d c_{c a t_{B^{\prime}}}}{d t}=r_{A \rightarrow B^{\prime}}-r_{A \rightarrow B}
$$

With

$$
r_{A \rightarrow B}=k_{A \rightarrow B} c_{c a t_{A}} \frac{P_{H_{2}}}{1+K_{H_{A}} P_{H_{2}}} ; r_{A \rightarrow B^{\prime}}=k_{A \rightarrow B^{\prime}} c_{\text {cat }_{A}} c_{c a t_{B^{\prime}}}
$$

while the concentration of inactivated species $c_{c a t}$ is

$$
\frac{d c_{c a t_{B d}}}{d t}=r_{B d}
$$

The kinetic equation for inactivation of species of type B is given by

$r_{B d}=k_{B_{d}} c_{c a t_{B}}$

Concentrations of all catalytic species are related through the mass balance $c_{\text {cat }_{C}}=c_{\text {cat }_{A}}^{0}-c_{\text {cat }_{A}}-c_{\text {cat }_{B}}-c_{\text {cat }_{B}}$, where $c_{\text {cat }_{A}}^{0}$ is the initial catalyst concentration. 
Transformations of reactant $\mathbf{1}$ follow the methylation pathway with competitive adsorption of hydrogen and the reactant in accordance to the Langmuir-Hinshelwood mechanism

$$
r_{B_{1 \rightarrow 2}}=\frac{k_{b 1} c_{1} c_{c a t_{B}} P_{H_{2}}}{\left(1+K_{a d s, b, 1} c_{1}+K_{H} P_{H_{2}}\right)^{2}}
$$

In eq. (9) $K_{H}$, which was considered to have a negligible temperature dependence, stands for hydrogen binding to the catalyst. The adsorption constant $K_{a d s, b, 1}$ accounts for adsorption of reactant 1 on the sites $\mathrm{B}$. The rate constant $k_{b 1}$ is a lumped one implicitly containing the adsorption constants.

In the kinetic model generation of side products from reactant 1 was taken into account through

$$
r_{B_{1 \rightarrow S}}=\frac{k_{b S} c_{1} c_{c a t_{B}}}{1+K_{a d s, b, 1} c_{1}+K_{H} P_{H_{2}}}
$$

Methylation of product $\mathbf{2}$ was assumed to follow exactly the same mechanism as substrate $\mathbf{1}$ with the same hydrogen pressure dependence in compliance with Figure 1d

$$
r_{B_{2 \rightarrow 3}}=\frac{k_{b 2} c_{2} c_{c a t_{B}} P_{H_{2}}}{\left(1+K_{a d s, b, 1} c_{1}+K_{H} P_{H_{2}}\right)^{2}}
$$

The apparent rate constants $k$ comprising adsorption coefficients to avoid correlation between parameters are temperature dependent according to the Arrhenius equation

$$
k=k_{0} e^{\frac{-E a}{R}\left(\frac{1}{T}-\frac{1}{\bar{T}}\right)}
$$

where the mean temperature $\bar{T}$ is in the current case $423 \mathrm{~K}$.

Component generation rates in a batch reactor can be easily written

$$
\frac{d c_{S}}{d t}=-r_{B_{1} \rightarrow S} ; \frac{d c_{1}}{d t}=-r_{B_{1 \rightarrow 2}}-r_{B_{1 \rightarrow S}} ; \frac{d c_{2}}{d t}=r_{B_{1 \rightarrow 2}}-r_{B_{2 \rightarrow 3}} ; \frac{d c_{3}}{d t}=-r_{B_{2 \rightarrow 3}}
$$

where $\mathrm{C}_{\mathrm{S}}$ is the concentration of side products.

\section{Comparison with experimental data}


Preliminary calculations demonstrated that the terms other than unity in denominators of eq. (9)-(11) can be neglected decreasing the number of parameters to be determined by numerical data fitting. The results of calculations given in Figure 5 show a very good correspondence between calculations and experimental data, which were treated simultaneously. The degree of explanation was $99.65 \%$.
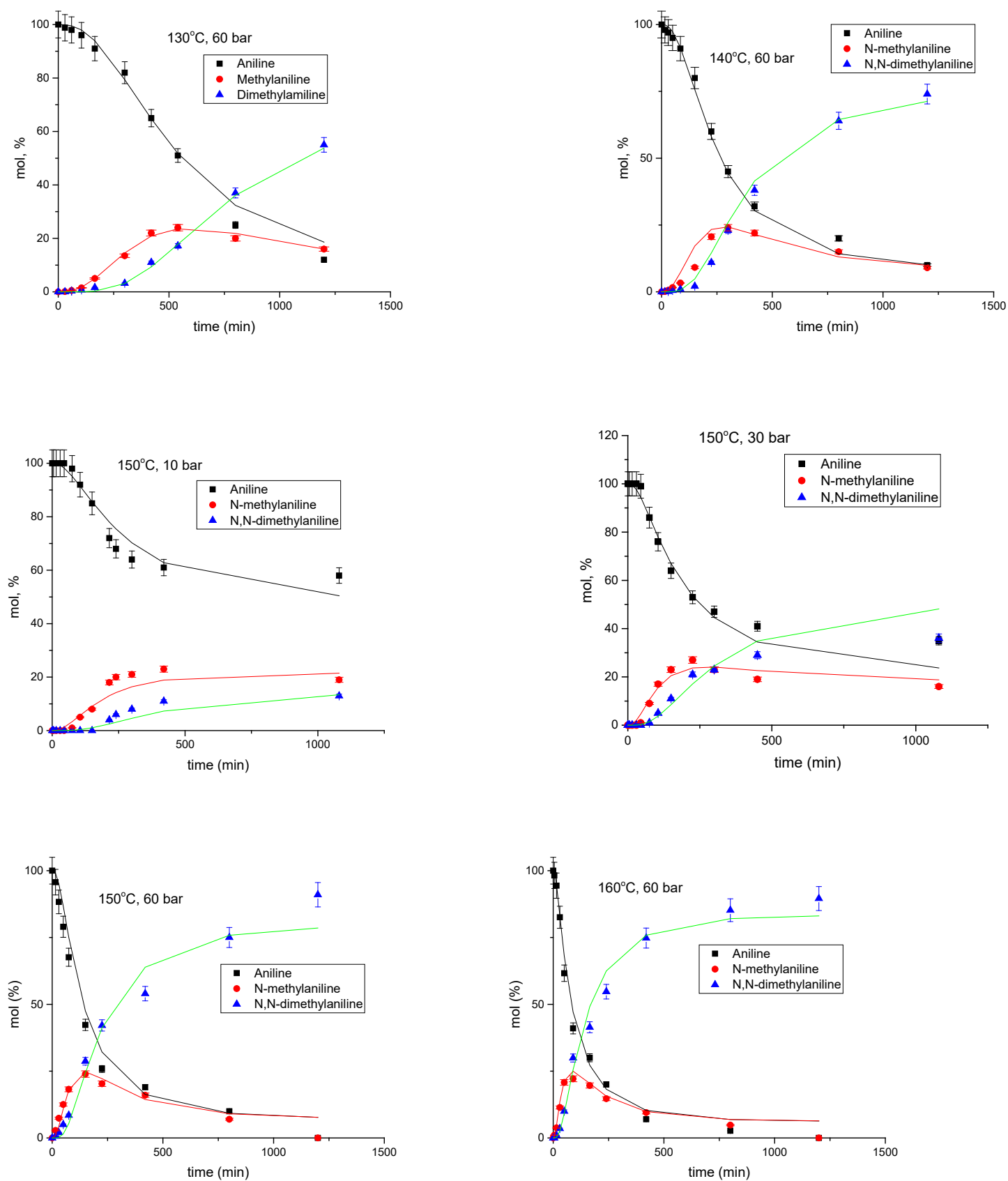

Figure 5. Kinetic profiles for the consumption of aniline $1(\mathrm{O})$ and formation of Nmethylaniline 2 (x) and N,N-dimethylaniline 3 ( ) as a function of reaction time with data sets 
in the N-methylation of aniline 1 using dimethyl carbonate ( 3 eq) and molecular hydrogen under different temperatures $\left(130-160^{\circ} \mathrm{C}\right)$ and pressures of molecular hydrogen (10-60 bar).

The values of kinetic parameters are given in Table 1.

Table 1. Values of kinetic parameters obtained in parameter estimation.

\begin{tabular}{|c|c|c|c|c|c|c|c|}
\hline Parameter & $\mathrm{k}_{\mathrm{B} 1->\mathrm{S}}$ & $\mathrm{k}_{\mathrm{B} 1->2}$ & $\mathrm{k}_{\mathrm{B} 2->3}$ & $\mathrm{k}_{\mathrm{Bd}}$ & $\mathrm{k}_{\mathrm{A}->\mathrm{B}}$ & $\mathrm{k}_{\mathrm{A}->\mathrm{B}}$ & $\mathrm{K}_{\mathrm{HA}}$ \\
\hline Value & $1.3 \times 10^{-5}$ & $3.2 \times 10^{-6}$ & $6.2 \times 10^{-6}$ & $5.1 \times 10^{-5}$ & $\begin{array}{r}1.43 \\
\times 10^{-3}\end{array}$ & $\begin{array}{l}4.33 \\
\times 10^{-5}\end{array}$ & 0.069 \\
\hline $\begin{array}{l}\text { Relative } \\
\text { standard } \\
\text { Error, \% }\end{array}$ & 15.6 & 13.0 & 13.6 & 8.4 & 26.3 & 22.0 & 33.9 \\
\hline Units & $\mathrm{s}^{-1}$ & $\mathrm{~s}^{-1}$ bar $^{-1}$ & $\mathrm{~s}^{-1} \mathrm{bar}^{-1}$ & $\mathrm{~s}^{-1}$ & $\mathrm{~s}^{-1}$ & $\mathrm{~s}^{-1}$ & bar $^{-1}$ \\
\hline Parameter & & $\mathrm{E}_{\mathrm{B} 1->2}$ & $\mathrm{E}_{\mathrm{B} 2->3}$ & $\mathrm{E}_{\mathrm{Bd}}$ & $\mathrm{E}_{\mathrm{A}->\mathrm{B}}$ & $\mathrm{E}_{\mathrm{A}->\mathrm{B}}$, & \\
\hline Value & Ca. 0 & 61.4 & 61.0 & 46 & 153 & 161 & \\
\hline $\begin{array}{c}\text { Standard } \\
\text { Error, } \%\end{array}$ & $>100 \%$ & 40.4 & 39.1 & 33.9 & 31.9 & 9.3 & \\
\hline Units & $\mathrm{kJ} / \mathrm{mol}$ & $\mathrm{kJ} / \mathrm{mol}$ & $\mathrm{kJ} / \mathrm{mol}$ & $\mathrm{kJ} / \mathrm{mol}$ & $\mathrm{kJ} / \mathrm{mol}$ & $\mathrm{kJ} / \mathrm{mol}$ & \\
\hline
\end{tabular}

The results show that the activation energies in consecutive transformations of reactant $\mathbf{1}$ to product $\mathbf{2}$ and subsequent transformation to product $\mathbf{3}$ are almost equal to each other, while the pre-exponential factor for formation of the product 3 is two-fold higher. This difference cannot be easily seen in kinetic curves due to interference of catalyst inactivation. Due to small amounts of the side products the value of the activation energy for their formation could not be determined reliably.

Visualization of the fractions of different types of catalytic species with time at different $\mathrm{T}$ is presented in Figure 6, clear showing that the rate of pressure independent interconversion between species A and B has a very strong temperature dependence. 

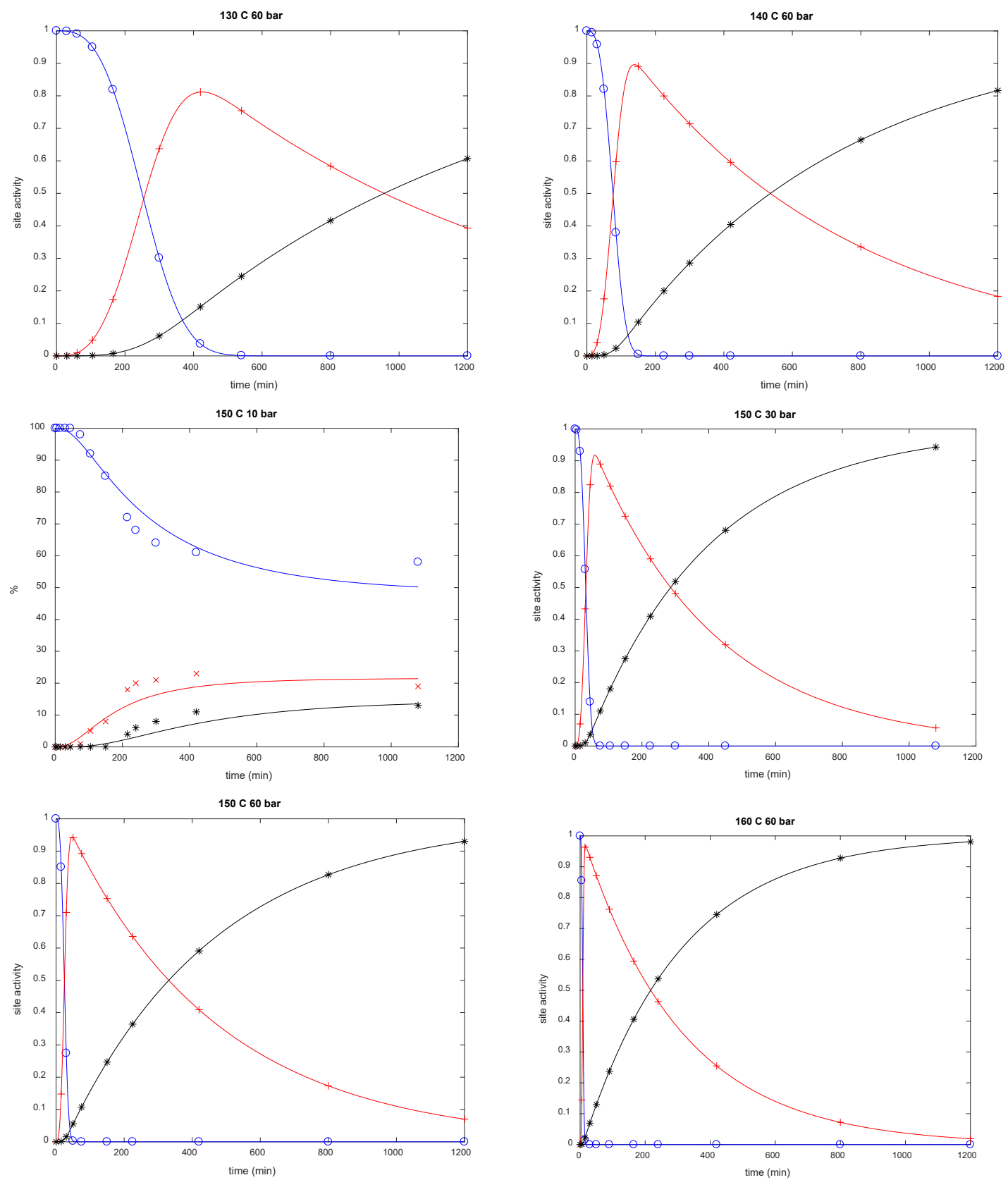

Figure 6. Relative fraction of catalytic species, blue (-o-) initial of A type, red (-+-) active of B type, black (-*) inactivated. 


\section{Conclusions}

Ruthenium-catalyzed reductive N-methylation of amines using dimethyl carbonate as $\mathrm{C} 1$ source was performed at $130-160^{\circ} \mathrm{C}$ exploring the influence of hydrogen pressure. At certain conditions, an induction period was observed, associated with low reaction rates. Such induction periods followed by rate acceleration were seen to be dependent on hydrogen pressure and were more pronounced at low temperature. Mechanistically presence of the induction period was explained by in-situ generation of active species. The concept of two sites was in line with structure -activity studies when N-methylation of aniline was done using its different para-substituted derivatives resulting in a non-linear Hammett plot with an upward concave behaviour.

Simultaneously with the acceleration catalyst deactivation was pronounced for experiments at lower hydrogen pressure (10, 30 bar).. A kinetic model has been proposed featuring transformation between different type of catalytic species More precisely the initial catalyst is transformed to the intermediate form further reacting with the starting catalytic species giving finally the catalytically active ones. Inactivation of the latter species during the reaction was also incorporated in the model.

In the kinetic model, transformations of aniline were considered to follow the methylation pathway with competitive adsorption of hydrogen and the reactant.

Kinetic modelling was done for all data sets together showing excellent correspondence between calculations and experiments. The results show that the activation energies in consecutive transformations of reactant aniline to N-methylaniline and subsequent transformation to $\mathrm{N}, \mathrm{N}$-dimethylaniline are almost equal to each other, while the pre-exponential factor for formation of the latter product is two-fold higher. 


\section{References}

1. M. Tsukamoto, K. Gopalaiah, H.B. Kagan, Equilibrium of homochiral oligomerization of a mixture of enantiomers. Its relevance to nonlinear effects in asymmetric catalysis, $\mathrm{J}$. Phys.Chem. B, 112 (2008) 112, 15361-15368.

2. D.G. Blackmond, Kinetic profiling of catalytic organic reactions as a mechanistic tool, J. Amer. Chem. Soc., 137 (2015) 10852-10866.

3. F.G. Helfferich, Kinetics of Homogeneous Multistep Reactions, in Comprehensive chemical kinetics, vol. 38. ed. R.G. Compton, G. Hancock, Elsevier, Amsterdam, 2001.

4. V. Leskovac, Comprehensive Enzyme Kinetics, Kluwer Academic/Plenum Publishers, New York, 2003.

5. O.N. Temkin, Homogeneous Catalysis with Metal Complexes: Kinetic Aspects and Mechanisms, Wiley, Weinheim, 2012.

6. D.Yu. Murzin, T. Salmi, Catalytic Kinetics, Chemistry and Engineering. 2d Edition, Elsevier, 2016.

7. T.O. Salmi, J.-P. Mikkola, J. Wärnå, Chemical Reaction Engineering and Reactor Technology, CRC Press, 2010.

8. H. S. Fogler, Elements of Chemical Reaction Engineering, third ed., Prentice Hall, NJ, 1998.

9. G.Marin, G. S. Yhablonsky, Kinetics of Chemical Reactions, Wiley, 2011.

10. F. Kapteijn, R.J. Berger, J A. Moulijn, Rate procurement and kinetic modelling, Handbook of Heterogeneous Catalysis, DOI: 10.1002/9783527610044.hetcat0092, Wiley, 2008.

11. J. R. Cabrero-Antonino, R. Adam, K. Junge, M. Beller, A general protocol for the reductive $\mathrm{N}$-methylation of amines using dimethyl carbonate and molecular hydrogen: mechanistic insights and kinetic studies, Catal. Sci. Tech., 6 (2016) 7956-7966.

12. S. A. Lawrence, Amines: Synthesis, Properties and Applications, Cambridge University Press, Cambrigde, 2006.

13. X. Ge, C. Luo, C. Qian, Z. Yu, X. Chen, Raney nickel catalyzed reductive N-methylation of amines with paraformaldehyde: theoretical and experimental study, RSC Adv., 4 (2014) 43195-43203.

14. T. Oku, Y. Arita, H. Tsuneki, T. Ikariya, Continuous chemoselective methylation of functionalized amines and diols with supercritical methanol over solid acid and acid-base bifunctional catalysts, . Am. Chem. Soc., 126 (2004) 7368-7377. 
15. Y. Cao, H. Cheng, L. Ma, F. Liu, Z. Liu, Research progress in the direct synthesis of dimethyl carbonate from $\mathrm{CO}_{2}$ and methanol, Catal. Surveys Asia, 16 (2012) 138-147.

16. E. Leino, P. Mäki-Arvela, V. Eta, D. Yu. Murzin, T. Salmi, J.-P. Mikkola, Appl. Catal. A. Gen., Conventional synthesis methods of short-chain dialkylcarbonates and novel production technology via direct route from alcohol and waste $\mathrm{CO}_{2}, 383$ (2010) 1-13.

17. E.Leino, P. Mäki-Arvela, V. Eta, N. Kumar, F. Demoisson, A. Samikannu, A.-R.Leino, A. Shchukarev, D. Yu. Murzin, J.-P. Mikkola, The influence of various synthesis methods on the catalytic activity of cerium oxide in one-pot synthesis of diethyl carbonate starting from CO2, ethanol and butylene oxide, Catal. Today, 210 (2013) 47-54.

18. P. Tundo, L. Rossi, A. Loris, Dimethyl carbonate as an ambident electrophile, J. Org. Chem., 70 (2005) 2219-2224.

19. Y. Ono, Catalysis in the production and reactions of dimethylcarbonate, an evironmentally benign building block, Appl. Catal., A, 155 (1997) 133-166.

20. J. Zheng, C. Darcel, J.-B. Sortais, Methylation of secondary amines with dialkylcarbonates and hydrosilanes catalyzed by iron complexes, Chem. Commun., 50 (2014) 14229-14232.

21. Y. Li, I. Sorribes, C. Vicent, K. Junge, M. Beller, Convenient reductive methylation of amines with carbonates at room temperature, Chem. - Eur. J., 21 (2015) 16759-16763.

22. S. Wesselbaum, T. vom Stein, J. Klankermayer, W. Leitner, Hydrogenation of carbon dioxide to methanol by using a homogeneous ruthenium-phosphine catalyst, Angew. Chem. Int. Ed., 51 (2012) 7499-7502.

23. I. Sorribes, J. R. Cabrero-Antonino, C. Vicent, K. Junge, M. Beller, Catalytic N-alkylation of amines using carboxylic acids and molecular hydrogen, J. Am. Chem. Soc. 137 (2015) $13580-13587$.

24. R. Adam, J. R. Cabrero-Antonino, K. Junge, R. Jackstell, M. Beller, Esters, including triglycerides, and hydrogen, as feedstocks for the ruthenium-catalyzed direct $\mathrm{N}$-alkylation of amines, Angew. Chem. Int. Ed., 55 (2016) 11049-11053.

25. S. Wesselbaum, V. Moha, M. Meuresch, S. Brosinski, K. M. Thenert, J. Kothe, T. v. Stein, U. Englert, M. Holscher, J. Klankermayer, W. Leitner, Hydrogenation of carbon dioxide to methanol using a homogeneous ruthenium-Triphos catalyst: from mechanistic investigations to multiphase catalysis, Chem. Sci., 6 (2015) 693-704.

26. H. Haario, ModEst, Parameter Estimation and Optimization Software, 2007, Helsinki.

27. S.R.Konuspaev,Kh.N.Zhanbekov,N.V.Kul'kova, D.Yu.Murzin, Kinetics of 4-tertbutylphenol hydrogenation over Rh, Chem.Eng.Technol., 20 (1997) 144-148. 
28. V. N. Sapunov, A. S. Stepacheva, E. M. Sulman, J. Wärnå, P. Mäki-Arvela, L. Zh. Nikoshvili, M.G. Sulman, A.I. Sidorov, B. D. Stein, D.Yu. Murzin, V.G. Matveeva, Stearic acid hydrodeoxygenation over Pd nanoparticles embedded in mesoporous hypercrosslinked polysterene, J. Ind. Eng. Chem,, 46 (2017) 426-435.

29. P. Subramaniam, J.J. S. J. Rose, R.J.E. Rathinakumari, A paradigm shift in the rate determining step from single electron transfer between phenylsulfinylacetic acids and iron(III) polypyridyl complexes to nucleophilic attack of water to the produced sulfoxide radical cation: a non-linear Hammett, J. Phys. Org. Chem., 29 (2016) 496-504.

30. D.G. Lee, L. N. Congson, Kinetics and mechanism of the oxidation of alcohols by ruthenate and perruthenate ions, Can. J. Chem., 68 (1990) 1774-1779.

31. S. Meenakshisundaram, R. Sockalingam, Nonlinear Hammett relationships in the reaction of peroxomonosulfate anion $\left(\mathrm{HOOSO}_{3}{ }^{-}\right)$with meta- and para-substituted anilines in alkaline medium, Collect. Czech. Chem. Commun. 66 (2001) 897-911.

32. A.K. Guha, C. K.Kim, H. W. Lee, Kinetics and mechanism of the pyridinolysis of N-arylP,P-diphenyl phosphinic amides in dimethyl sulfoxide, J. Phys. Org. Chem., 24 (2011) 474479.

33. P. Subramaniam, S. Sugirtha Devi, S. Anbarasan, Proximal effects of the nitrogen bases in the oxidative decarboxylation of phenylsulfinylacetic acids by (oxo)salenchromium(V) complexes, J. Molec. Catal. A. Chem., 390 (2014) 159-168.

34. K.K. Adhikary, H.W. Lee, Bull. Korean Chem. Soc., Significant substituent effects on pyridinolysis of aryl ethyl chlorophosphates in acetonitrile, 35 (2014) 1460-1464.

35. P. Subramaniam, S. Anbarasan, S. Sugirtha Devi, A. Ramdass, Modulation of catalytic activity by ligand oxides in the sulfoxidation of phenylmercaptoacetic acids by oxo(salen)chromium(V) complexes, Polyhedron, 119 (2016) 14-22.

36. O. Exner, The Hammett equation-the present position, in N. B. Chapman, J. Shorter (Eds.), Advances in Linear Free Energy Relationships, Plenum Press, London and NY, 1972, pp. 1- 69 .

37. D.Yu. Murzin, Interpretaion of rate optima vs reaction parameters in steady state catalytic kinetics: molecular aspects beyond concentration dependences , Molec.Catal, 433 (2017) $321-333$

38. V.P. Ananikov, I.P. Beletskaya, Towards an ideal catalyst from atomic centers to a “cocktail” of catalysts, Organometallics, 31 (2012) 1595-1604.

39. R. G. Konsler, J. Karl, E.N. Jacobsen, Cooperative asymmetric catalysis with dimeric salen complexes, J. Amer. Chem. Soc., 120 (1998) 10780-10781. 
40. T. Rosner, J. Le Bars, A. Pfaltz, D. G. Blackmond, Kinetic studies of Heck coupling reactions using palladacycle catalysts: experimental and kinetic modeling of the role of dimeric species, J. Amer. Chem. Soc., 123 () 1848-1855.

41. M. E. Noble-Teran, T. Buhse, J.-M. Cruz, C. Coudret, J.-C. Micheau, Nonlinear effects in asymmetric synthesis: a practical tool for the discrimination between monomer and dimer catalysis, ChemCatChem, 8 (2016) 1836-1845. 